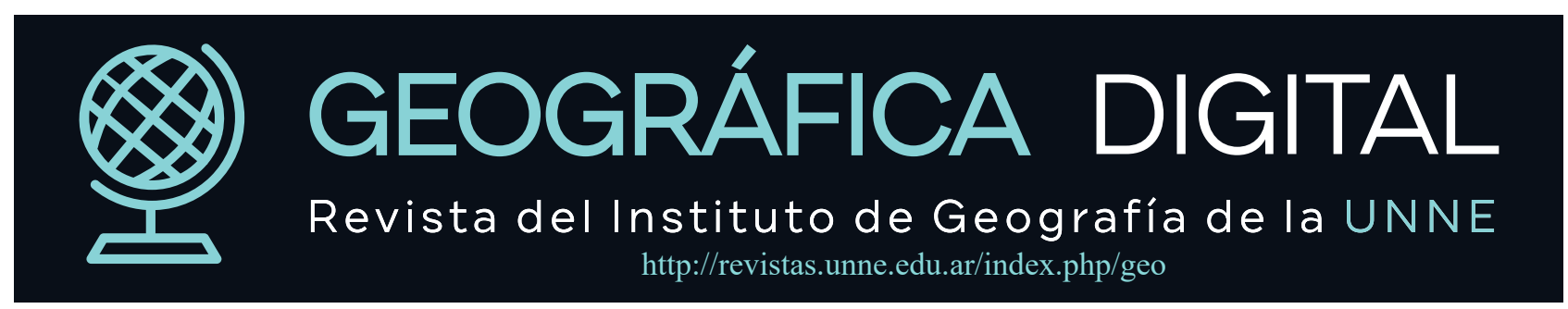

Revista Geográfica Digital, 2021, Vol. 18, No 35, 51-61 pp., E-ISSN: 1668-5180: DOI: http://dx.doi.org/10.30972/geo.18355133

\title{
Google Drive como facilitador de comunidades pedagógicas. Experien- cia de formación continua en Didáctica de la Geografía
}

\section{Google Drive as a facilitator of pedagogical communities. Continuing education experience in Didactics of Geography}

\section{Acosta, Melina I. ${ }^{1}$; Nin, María C. ${ }^{2}$}

1UNLPam. Instituto de Geografía, Facultad de Ciencias Humanas, Universidad Nacional de La Pampa. meliacosta24@gmail.com ${ }^{2}$ Universidad Nacional del Sur. Facultad de Ciencias Humanas, Universidad Nacional de La Pampa. ninmcristina@gmail.com

\footnotetext{
R e s u m e n

Palabras claves:

Geografía

Formación

Tecnología

Comunidad Pedagógica

En la actualidad existe consenso social acerca del uso de las tecnologías de la información y la comunicación, es por ello que los ámbitos de formación profesional, en este caso de formación docente específica, es preciso implementar Tecnologías de la Información y la Comunicación (TIC) para fortalecer el trabajo colaborativo. En el presente artículo se expone un proyecto de trabajo de la disciplina Didáctica Especial de la Geografía, Facultad de Ciencias Humanas, Universidad Nacional de La Pampa (UNLPam) que utiliza el Google Drive como dispositivo tecnológico. La experiencia comienza en el cursado de la materia correspondiente al cuarto año del Profesorado en Geografía y pretende tener continuidad y ubicuidad más allá de los tiempos curriculares de un cuatrimestre del Plan de Estudios. Estimular la conformación de equipos de enseñanza para construir un colectivo docente que participe activamente en la construcción de comunidades pedagógicas, con el fin de mejorar las prácticas educativas, compartir actualizaciones académicas y garantizar aprendizajes en la aplicación de herramientas que servirán como insumos para los futuros profesores.
}

Key words:

Geography

Training

Technology

Pedagogical Community

\section{A b s t r a c t}

Currently there is a social consensus about the use of information and communication technologies that is why the areas of professional training, in this case of specific teacher training, it is necessary to implement Information and Communication Technologies (ICT) to strengthen collaborative work. In this article, a work project of the Special Didactic discipline of Geography, Faculty of Human Sciences, National University of La Pampa (UNLPam) that uses Google Drive as a technological device is exposed. The experience begins in the course of the subject corresponding to the fourth year of the Geography Faculty and aims to have continuity and ubiquity beyond the curricular times of a semester of the Study Plan. Encourage the formation of teaching teams to build a teaching collective that actively participates in the construction of pedagogical communities in order to improve educational practices, share academic updates and be able to guarantee learning through the application of tools that will serve as inputs for future teachers. 


\section{Introducción}

Las prácticas de enseñanza innovadoras implican que el docente reconozca la necesidad de generarlas de manera genuina, y para lograr que sean significativas deberían responder al contexto educacional con el propósito de mejorar la relación de la enseñanza con los aprendizajes a partir de propiciar entornos virtuales. La innovación educativa es entendida según la Comisión Económica para América Latina y El Caribe (CEPAL, 2008) como:
(...) el cruce entre diversos procesos, donde la teoría se encuentra con la práctica, donde los innovadores intercambian su experiencia, los patrocinadores financian y arriesgan, las organizaciones públicas y privadas cooperan, el conocimiento científico se complementa con el tradicional, que proviene de la propia experiencia, y la necesidad práctica se encuentra con la oferta de conocimiento aplicable (p.34).

El saber y las producciones de origen tecnológico están instalados en la vida cotidiana de la sociedad del siglo XXI y por ende deben formar parte de la enseñanza. Los estudiantes que dispongan de dispositivos tecnológicos para aprender desarrollarán habilidades tanto individuales, como cognitivas y de interacción y trabajo en equipo. Es por ello que, en la formación del Profesorado, en este caso de Geografía, resulta innovador e imprescindible incorporar en las prácticas de enseñanza la creación de espacios mediados por el uso de la tecnología para propiciar comunidades de conocimiento como formas de producir y circular saberes. Tal como expresa Maggio (2012): “(...) las prácticas de la enseñanza ya no pueden ser lo que fueron y podríamos abocarnos a su recreación y, por lo tanto, a la reinvención del campo de la tecnología educativa" (p.24). En este sentido Castells (2008) planteaba que vivimos en la era de la información. Reconocernos en este contexto ser partícipes activos del uso de tecnologías tanto en el desempeño profesional como en la vida cotidiana es una capacidad o competencia que hay que enseñar.

Formar a los estudiantes, futuros profesores en Geografía desde una perspectiva integral, es decir, tanto desde una alfabetización tradicional como desde las tecnologías de la información y comunicación, constituye un desafío para los formadores.

Las TIC conforman aquel conjunto de tecnologías, herramientas, vías o canales que permiten adquirir, acceder, obtener, almacenar, procesar, registrar, compartir y transmitir información digitalizada, en diversidad de códigos y formas, mediante la combinación de texto, imágenes y sonidos (Murillo, 2011).

En el campo educativo, el desarrollo profesional implica que los equipos docentes muestren intenciones de prepararse para tomar decisiones, explorar nuevas estrategias de trabajo y resolver problemas emergentes con el propósito de reducir la brecha cultural y generacional con los/as estudiantes. Manso, Pérez, Libedinsky, Light y Garzón (2011) expresan que la profesionalización docente es:
un proceso por el cual los docentes adquieren y/o desarrollan capacidades con el propósito de implementar nuevas estrategias de enseñanza (...) proceso que les permite reconocer nuevas posibilidades, adquirir la confianza y la autonomía necesarias para poner en práctica nuevas propuestas e, incluso, experimentar nuevos modos de posicionarse respecto a la enseñanza y el aprendizaje, y aún de cambiar sus creencias (p.78).

La posibilidad de ofrecer a los estudiantes del profesorado instrumentos de enseñanza basados en la inclusión educativa y digital implica asumir, desde la formación, un enfoque disciplinar basado en las oportunidades y estrategias innovadoras que invitan a problematizar y diversificar las estrategias formativas. Maggio (2012) sostiene que la inclusión educativa genuina alcanza los propósitos de la enseñanza y sus contenidos, pero adquiere su mejor expresión en la propuesta didáctica cuando lucha en este plano de la práctica el entramado de los desarrollos tecnológicos en los procesos de producción del conocimiento en el campo al que esté referenciando sobre las tramas de construcción del conocimiento disciplinar en la contemporaneidad.

A partir de allí es que, la enseñanza poderosa, en palabras de Maggio (2012), crea y (re)crea una propuesta original que nos transforma como sujetos y cuyas huellas permanecen al paso del tiempo. "La enseñanza poderosa entonces: da cuenta de un abordaje teórico actual; permite pensar el modo de la disciplina; mira en perspectiva; está formulada en tiempo presente; ofrece una estructura que en sí es original y conmueve y perdura" (Maggio, 2012, p.62).

El objetivo del presente artículo es explicar una experiencia desarrollada en la formación docente del profesorado en Geografía en la asignatura Didáctica Especial de la Geografía, de la UNLPam a partir de un dispositivo de enseñanza mediado por la tecnología. Litwin (1993 en Litwin 2005, p.26) entiende a la "tecnología educativa como el cuerpo de conocimientos que, basándose en disciplinas científicas referidas 
a las prácticas de enseñanza, incorpora todos los medios a su alcance y responde a la consecución de fines en los contextos socio-históricos que le otorgan significación". De este modo, las prácticas de enseñanza a partir del uso de tecnologías se han instalado desde hace varias décadas, aunque se reconocen resistencias por parte del colectivo docente en incorporarlas de manera habitual en las aulas. Respecto a este tema, se debe dar un gran debate en relación a los Programas de las asignaturas, que de modo transversal debiéramos implementar las TIC en las cátedras, sobre todo siendo formadores de formadores.

\section{Organización de la asignatura Didáctica de la Geografía}

La cátedra Didáctica Especial de la Geografía, ubicada en el cuarto año del segundo cuatrimestre, integra la teoría y la práctica de los contenidos propiamente disciplinares y los pedagógico-didácticos. Es durante el cursado de esta materia que los/as estudiantes aplican la teoría aprendida en las materias específicas y en las relacionadas con las asignaturas de la formación docente, para comenzar su tarea como profesionales de la enseñanza.

Los conocimientos imprescindibles que deben estar presentes en la formación de un profesor competente y crítico corresponden a los específicos de la materia que va a enseñar, el conocimiento pedagógico sobre la organización y funcionamiento del aula y de las instituciones educativas, el conocimiento del currículo, de los alumnos y del contexto educativo. Sin dudas uno de los más relevantes es el conocimiento pedagógico, identificado en la Didáctica Especial. Tal como expresa Benejam (2001):

El hecho de enseñar algo a alguien, exige que el profesor tome una serie de decisiones (sobre el contenido, el método, las estrategias de aprendizaje, los materiales, entre otros). La Didáctica tiene por objeto que el enseñante decida de manera consciente, responsable y pertinente. Para ello la didáctica indaga en el estudio de la teoría y en el contexto práctico donde opera (p.62).

Las palabras de la autora reflejan la necesidad de que los/as futuros/as profesores/as tengan una continuidad en su formación. Esto es, profundizar el trabajo iniciado en las asignaturas propias de la formación docente tales como Psicología, Pedagogía, Didáctica, Política y Legislación Escolar, para poner en juego dichos contenidos con los propios de la didáctica específica. Camino que tendrá su correlato y que se concretará en la práctica educativa durante Residencia Docente. Respecto al Programa de la asignatura, los ejes responden a la idea de formación de un profesor crítico, autónomo, reflexivo, que conozca el contexto legal, institucional y áulico en el que se van a desempeñar. Para ello la propuesta de la cátedra consta de cinco ejes de los cuales el primero "Geografía científica contemporánea y Geografía escolar" pretende recuperar saberes trabajados en materias y años previos tales como Introducción a la Geografía y Epistemología con el propósito de articularlos y comenzar el camino de pensar en su enseñanza.

Con el segundo eje "Marco normativo y curricular de la Educación Secundaria y la enseñanza de la Geografía”, se analiza la Ley de Educación Nacional (LEN) № 26.206/06 y los marcos normativos que sustentan la organización de las instituciones secundarias, la evolución desde la elaboración de los Núcleos de Aprendizaje Prioritarios (NAP), hasta la incorporación de los Materiales Curriculares elaborada en la provincia de La Pampa. En tal sentido, las propuestas de cambio y transformación curricular estimulan la reflexión sobre la didáctica de la geografía, promueven el debate, pero para que ello se efectivice con compromiso es preciso que los/as profesores/as reconozcan los marcos legales y se analicen las fuentes primarias donde se expresan las finalidades de la educación secundaria obligatoria; y de este modo se interpreten y comprendan los aportes que la geografía puede realizar a la enseñanza (Souto González, 1999).

En el tercer eje se propone conocer y analizar los "Proyectos transversales a la enseñanza de la Geografía" que incorpora en la política educativa el Ministerio de Educación tanto nacional como provincial. Para ello se indaga en los programas transversales que las instituciones educativas ponen en marcha, tales como Educación Sexual Integral (ESI), Educación y Memoria, Programa Conectar Igualdad ${ }^{1}$, Plan de Mejora Institucional, Políticas del Cuidado, entre otros. En este eje también se mencionan tareas inherentes al trabajo de un/a profesor/a tales como los actos escolares, la participación en jornadas institucionales, el trabajo en equipos de enseñanza y proyectos institucionales e interdisciplinarios. Los saberes a trabajar en este núcleo temático son significativos ya que todo/a profesor/a no solo enseña la disciplina, sino que las 1 El Programa Conectar Igualdad consistió en la entrega de cinco millones de netbooks a estudiantes y docentes de establecimientos públicos de educación secundaria, especial y de formación docente, en un plazo aproximado de tres años. Asimismo, se desarrollaron contenidos digitales como propuestas didácticas con el propósito de establecer la inclusión digital. El ensamblaje de las computadoras se realizaba en la provincia más austral de Argentina, Tierra del Fuego. El financiamiento de este programa proviene de la reasignación de partidas presupuestarias del Presupuesto Nacional. Las inversiones también consistieron en instalar antenas para proveer internet inalámbrico a las instituciones educativas del país, capacitar a los docentes y referentes tecnológicos en cada colegio, escuela e instituto de formación docente. 
tareas que se desarrollan en las instituciones y en el aula, son transversales a todas las disciplinas y resulta pertinente incluir este tipo de programas y proyectos, con el fin de otorgar a los/as estudiantes mayor conocimiento acerca del trabajo docente en cuanto a sus roles y funciones.

En "Enseñanza de la Geografía", cuarto eje, se pretende que los/as estudiantes se apropien de la metodología didáctica y las estrategias para enseñar Geografía. Elegir el camino adecuado para alcanzar las metas de enseñanza pensadas es la tarea de un/a profesor/a que debe tomar decisiones como recortar, secuenciar, seleccionar contenidos y conceptos clave frente al currículo, decidir acerca de los procesos explicativos que va a planificar para que sus estudiantes aprendan de manera significativa actividades, recursos y estrategias. Es decir que en este eje se ponen en juego saberes ya estudiados como las teorías constructivistas y críticas de la enseñanza con saberes que resulten novedosos para la enseñanza de la Geografía. Es en esta instancia de la cursada donde los/as estudiantes realizan observaciones de clases en escuelas secundarias. Por lo tanto, ya sea en las observaciones como en las clases donde se las analiza comienza una interpretación dialéctica entre la teoría-praxis y es esta experiencia la que les posibilita pensar y construir su propio perfil docente partiendo del principio de acción y reflexión para la acción. En el último eje "La evaluación como parte del proceso de enseñanza" se pretende realizar un recorrido teórico por las diferentes lógicas evaluativas e instrumentos de evaluación para establecer relación directa con sus experiencias personales como sujetos evaluados y la normativa de evaluación vigente en la Provincia de La $\mathrm{Pampa}^{2}$. Asimismo, se analizan y construyen rúbricas, valoraciones pedagógicas, protocolos de seguimiento y acompañamiento a las trayectorias educativas, listas de cotejo de saberes.

La propuesta que se presenta a continuación constituye una estrategia metodológica, didáctica y tecnológica que refuerza el eje abordado en la asignatura sobre los proyectos transversales, en este caso a partir de la implementación de las tecnologías. Este eje, en este sentido tiene un doble propósito, como se mencionó anteriormente presta especial atención en generar enseñanzas que enriquezcan las clases de Geografía en Educación Secundaria, como así también pretende atender y acercar las demandas de los/as estudiantes de manera inmediata y permanecer comunicados/os entre todos/as los/as integrantes de la comunidad digital.

\section{Metodología}

El problema identificado es que a lo/as estudiantes que cursan y aprueban la asignatura Didáctica Especial de la Geografía se les dificulta sostener en el tiempo la conexión y el vínculo educativo con la Facultad de Ciencias Humanas y, en general, la mayoría son estudiantes del interior de la provincia que regresan a sus localidades, y se complejiza el regreso a la Universidad cuando son graduados. Asimismo, asisten de manera esporádica a realizar actividades que propone la Universidad Nacional de La Pampa ${ }^{3}$ como seminarios, capacitaciones, cursos, charlas, entre otros/as. La Universidad ofrece la posibilidad de que las cátedras puedan migrar información a la plataforma del Campus Virtual que posee institucionalmente, aunque una vez que aprueban la asignatura esos materiales carecen de accesibilidad.

En este sentido, la propuesta pretende interactuar a través del espacio virtual con estudiantes y graduados/as para ampliar los lazos de comunicación y compartir experiencias de prácticas y enseñanzas profesionales, actualizaciones académicas, recursos, entre otras/os, que favorezcan una red sostén de formación continua a partir de imprimirle un valor pedagógico al trabajo colaborativo con de las TIC.

Este proyecto está pensado y diseñado para llevar adelante con los/as estudiantes que cursan la asignatura Didáctica Especial de la Geografía y, a su vez generar continuidad y contigüidad en la comunicación y en los aprendizajes de estudiantes con las docentes a cargo de la asignatura y los/as estudiantes de los años subsiguientes. La metodología es incorporar año a año a los/as que se incorporan a la asignatura para formar una comunidad pedagógica y virtual de enseñanzas y aprendizajes que se sustentan en un proceso de formación permanente y acompañarlos/as en el ejercicio de la formación

\footnotetext{
2 Resolución ME N. 701/20 que reemplaza por el tiempo de Pandemia a la Resolución MCE № 660/13 y la Disposición de Evaluación ME N. 165/18.

3 La UNLPam a través del Área de Tecnologías Educativas, desde 2017 cuenta con un Programa de Virtualización de Actividades Curriculares que tiene como finalidad lograr un mayor compromiso, por parte de los docentes, con el uso de herramientas tecnológicas y la educación a distancia. El mismo se enmarca dentro del Contrato Programa Integral MEYD-UNIV-NAC-DE LA PAMPA (RE-2017-14823551-APN-DD\#ME) y este a su vez, en los lineamientos del Plan Estratégico 2016-2020 (RCS. 402/16). El Programa pretende promover la construcción de 200 propuestas mediadas tecnológicamente en el contexto actual, en el que las tecnologías digitales flexibilizan tiempos y espacios distanciados y ofrecen alternativas potentes para aumentar, enriquecer y transformar la enseñanza complementando las prácticas presenciales. Las mismas, se distribuirán en cuatro convocatorias entre 2017-2020. Asimismo, se cuenta con una Plataforma de Campus Virtual en la que pueden virtualizar entre un 65\% o 100\% las cátedras de las diferentes Facultades de la UNLPam. Como es un proceso gradual, no todas las cátedras han podido virtualizar sus contenidos, como es el caso de Didáctica Especial de la Geografía.
} 
continua en la práctica docente.

Los objetivos son crear un espacio virtual de comunicación para ampliar o re-localizar (MartínBarbero, 2002) los tiempos y lugares de intercambios de saberes (ubicuidad); compartir e intercambiar información académica como libros escolares y académicos, artículos de divulgación científica, normativas, textos de interés, propuestas didácticas, entre otros/as; fomentar la formación de equipos pedagógicos/ comunidades pedagógicas a partir del diálogo interpersonal y en red y elaborar documentos compartidos que enriquezcan los aprendizajes colaborativos en ambientes y entornos virtuales. La integración didáctica de las TIC implica reorganizar los saberes y las relaciones de autoridad pedagógica. Este enfoque plantea que a partir de la cultura digital, se desarrollen nuevas formas de aprender y de enseñar basadas en la legitimidad de los conocimientos a partir del diálogo y el debate intergeneracional. Durante el 2020 se profundiza la necesidad de mantener el vínculo pedagógico con las/as estudiantes,

las plataformas virtuales, surgieron o (re)surgieron como novedosas ante esta situación de aislamiento social. Se modifica la noción de escala, el espacio ahora cotidiano, doméstico, simbólico, un rincón de nuestros hogares se convierte en lugar de encuentro virtual, se abren ventanas que nos conectan con las instituciones educativas de los diferentes niveles del sistema educativo (Nin, Acosta y Leduc, 2020, p.226).

El tiempo y el espacio de los aprendizajes cobran nuevos sentidos y significados a partir de la coconstrucción con otros de modo asincrónico.

Las acciones que se desarrollan corresponden a organizar un Google Drive acorde con los ejes propuestos en la asignatura y actualizarlo de manera permanente; crear una portfolio de recursos didácticos en la que todos los/as integrantes de la comunidad pedagógica puedan aportar al conocimiento colectivo; incorporar de manera continua materiales correspondientes a la asignatura y a la carpeta de banco de recursos didácticos a modo de retroalimentar la actualización profesional y propiciar intercambios entre los/as participantes.

La organización en Google Drive de la asignatura surge a partir del problema mencionado anteriormente, a su vez, es una iniciativa de la cátedra para los/as estudiantes, aunque se construye con los aportes de las profesoras como así también, de todos los estudiantes y graduados que necesitan incorporar recursos, información, estrategias y actualización académica para el desarrollo de sus actividades áulicas en instituciones secundarias. Además, se establece un nuevo vínculo docentes-estudiantes-graduados desde la noción de mediación a partir de las tecnologías y como una nueva forma de convivencia intergeneracional. Es decir, este proyecto requiere de un verdadero trabajo en equipo para lograr una auténtica integración de las tecnologías interactivas en la formación académica.

Desde el momento que se organiza, se piensa en un proceso extendido en el tiempo y en el espacio, la idea es ir sumando a graduados/as que necesitan y requieren acercarse de alguna manera a la Universidad, que debido a su trabajo en las instituciones de educación secundaria se encuentran distanciados de las actividades de perfeccionamiento que propone la Facultad de Ciencias Humanas. De este modo, incorporan conocimientos y relaciones pedagógicas virtuales desde la ubicuidad y la autorregulación de saberes como forma de actualización académica.

\section{Promover la creación de una comunidad pedagógica a través del espacio virtual}

La evolución de las tecnologías de la información y comunicación, conocidas como TIC, es una realidad, por lo tanto, para que la educación se adapte a las exigencias de este nuevo paradigma de ciudadanía digital, el sistema educativo debería estimular el uso de innovaciones tecnológicas. En este contexto los/as docentes tienen que desarrollar una praxis con carácter innovadora, es decir adquirir un conocimiento sobre el manejo y enseñanza de la tecnología como una competencia digital (Pozo Sánchez, López Belmonte, Moreno Guerrero e Hinojo Lucena, 2020). En relación al término competencia, se entiende como “(...) aquel conjunto integrado y dinámico de saberes, habilidades, capacidades y destrezas, actitudes y valores puestos en juego en la toma de decisiones, en la acción-en el desempeño concreto-del sujeto en un determinado espacio (profesional, laboral, etc.)" (Coronado, 2013, p.19). La autora considera tres tipos de competencias: generales, específicas y transversales; el uso de tecnologías de la información y comunicación es una competencia general debido a que se espera que cualquier graduado de educación superior las pueda desarrollar. Por su parte Perrenoud en su libro "Diez Nuevas Competencias para enseñar" publicado en 1999 en francés, consideraba al manejo y uso de las nuevas tecnologías como una de ellas. En relación al uso de nuevas tecnologías en la enseñanza expresa que “(...) pueden reforzar la contribución de los trabajos pedagógicos y didácticos contemporáneos, puesto que permiten crear situaciones de 
aprendizajes enriquecedoras, complejas, diversificadas (...)" (Perrenoud, 2007, p.119).

Entre los elementos que se requieren para que el sistema educativo implemente el uso adecuado de las TIC de manera eficaz se pueden identificar; las destrezas en el uso de las tecnologías, el apoyo a nivel de infraestructura y formación por parte de la administración educativa, el uso positivo de las mismas y resultados positivos en el proceso de enseñanza y de aprendizaje (Moreira, García, Conde y González, 2019 en Pozo Sánchez et al, 2020). Según Poggi (2007), el uso e incorporación de las TIC en la educación está ligada a las políticas de igualdad por lo tanto ese uso debe ser estratégicamente planificado. Asimismo, expresa que "es fundamental el papel del Estado que aspire a poner al alcance de todos los ciudadanos un uso productivo y crítico de estos nuevos productos culturales" (Poggi, 2007, p.17). En este contexto, Tedesco (2007) reconoce en los docentes o en los equipos docentes a los/as actores clave para la incorporación de tecnologías en las aulas, es decir, que las políticas educativas tienen que planificar estratégicamente la formación del colectivo profesional docente para que exista un cambio real en las aulas. En este caso en Argentina, la formación docente en TIC, significó garantizar políticas educativas en materia de inclusión digital. En este marco, se realizaron capacitaciones y especializaciones en el uso de herramientas tecnológicas a docentes de escuelas y colegios públicos, acompañadas del Programa Conectar Igualdad ${ }^{4}$ en el año 2010, que mostró un período de auge durante los años que fueron entregadas las netbooks tanto a docentes como a estudiantes. Al mismo tiempo, se llevaron a cabo capacitaciones dirigidas a los docentes como la Especialización en Educación y TIC dictada por el INFOD. Por otro lado, se comenzó a evidenciar un período de declinación para el año 2015 y ya en 2016 comenzó a languidecer hasta desaparecer (Acosta y Pérez 2019, p.78).

Los cambios tecnológicos que se han desarrollado desde los últimos años han creado la necesidad de formar estudiantes, y en nuestro caso, futuros profesores desde una perspectiva integral desde lo disciplinar, pedagógico-didáctico y tecnológico. Es decir teniendo en cuenta la alfabetización tradicional como desde las emergentes Tecnologías de la Información y Comunicación (TIC). La naturaleza del saber ha cambiado debido a las TIC. Esta cuestión ya fue señalada por Lyotard (1994) quien, sobre finales de la década del setenta, anticipó: "el saber se encuentra o se encontrará afectado en dos principales funciones: la investigación y la trasmisión de conocimientos (...). Los "productores» del saber, lo mismo que sus utilizadores, deben y deberán poseer los medios de traducir a esos lenguajes lo que buscan, los unos al inventar, los otros al aprender" (p.14). Es decir que la tecnología promueve una reinterpretación de los roles de los diferentes actores educativos.

El contexto actual no está caracterizado por el desplazamiento sino por la convergencia. Se sostiene que estamos asistiendo al borramiento de límites, a la fusión de tecnologías, formas y prácticas culturales que antes eran independientes, tanto en el punto de producción como en la recepción. Sin duda, esta convergencia es en parte el resultado de los cambios operados en el terreno de la tecnología. La posibilidad de "digitalizar" toda una variedad de formas diferentes de comunicación (no solo escritura, sino también imágenes visuales y en movimiento, música, sonido y habla) transforma la computadora en mucho más que una calculadora o una máquina de escribir con memoria: la convierte en un medio que permite proporcionar y producir no sólo textos escritos, sino textos en una variedad de formatos $y$, como resultado, la pantalla digital ha devenido punto focal de toda una variedad de opciones de entretenimiento, información y comunicación (Buckingham, 2008, p.110).

La Universidad, como centro de profesionalización, tiene la responsabilidad pedagógica de formar no solo como espectadores y consumidores sino también como usuarios activos, creadores y generadores de la Web 2.0 para el desempeño académico y laboral de los futuros profesionales. En este contexto el reto de la formación universitaria es estimular y crear condiciones para el trabajo colaborativo o cooperativo. Los docentes desde nuestro desempeño en las asignaturas asumimos la responsabilidad de transmitir aprendizajes significativos y colaborativos en equipos interpersonales y en ámbitos virtuales. Los fundamentos teóricos se encuentran además, en la teoría de aprendizaje constructivista y en el conectivismo. En esta última:

El aprendizaje es un proceso que ocurre al interior de ambientes difusos de elementos centrales cambiantes, que no están por completo bajo control del individuo. El aprendizaje, definido como conocimiento aplicable, puede residir fuera de nosotros -al interior de una organización o una base de datos-, está enfocado en conectar conjuntos de información especializada, y las conexiones que nos permiten aprender

$4 \quad$ En el año 2018 el Programa asume el nombre Aprender Conectados, aunque sin entrega de computadoras y algunas provincias no pudieron sostenerlo. A partir del cambio de gobierno en el 2019, se confirma el regreso de Conectar Igualdad, que será lanzado por el Ministro de Educación de la Nación. En el contexto de Pandemia del COVID-19, se comienzan a distribuir netbooks para sostener la continuidad pedagógica y favorecer el acceso tecnológico a niños, niñas y adolescentes. 
más tienen mayor importancia que nuestro estado actual de conocimiento (Siemens, 2004, p.6).

Es decir, que el aprendizaje ya no está dado exclusivamente por actividades individuales o aisladas, sino que ocurre por la interacción con otros y es mediado ineludiblemente por las TIC.

Para lograr propuestas que prioricen la mejora de las prácticas de enseñanza y a su vez, provoquen un impacto relevante en los aprendizajes, es preciso organizarse en equipos de trabajo, contar con un proyecto curricular que fundamente teóricamente dicha innovación. El profesor aislado en el aula, sin poder contrastar sus análisis empíricos del aprendizaje en el aula, forma parte del pasado educativo. En el mundo global, lleno de flujos de informaciones, es preciso seleccionar datos, compararlos con las exigencias de otros lugares, verificar pruebas, validar la bibliografía, difundir los conocimientos, abrirse a los problemas de la comunidad implica establecer como prioridad el desempeño de equipos a partir de comunidades pedagógicas para lograr integralidad disciplinar (Nin y Acosta, 2020; Nin, Leduc y Acosta, 2020). "Así, hay una potencialidad en las prácticas formativas en las que profesores colectivamente cuestionan la realidad que viven y piensan en estrategias para enfrentar sus dificultades con la finalidad de lograr aprendizajes significativos con relación al desarrollo del pensamiento geográfico" (de Souza Cavalcanti y Dos Santos, 2020, p.26).

Generar una propuesta de enseñanza que proponga el diálogo y que tenga como propósito la construcción colectiva en un contexto educativo incorpora oportunidades pedagógicas para formarse como equipo de enseñanza. La construcción colectiva, se constituye al mismo tiempo en desafío. Producir un espacio virtual educativo que se sostenga en el tiempo extra curricular, que estimule procesos enriquecedores y profundos y que provoque una enseñanza relevante.

En el presente proyecto se pretende aplicar las posibilidades de la herramienta Google Drive, una de las herramientas que ofrece Google Apps para trabajar de manera colaborativa y online con el propósito de sostener la comunicación con los grupos de estudiantes que avanzan en la carrera y se convierten en Profesores. Esta herramienta es simple, de fácil accesibilidad, habilita el acceso a información, no necesita de grandes conocimientos informáticos y potencia la comunicación entre profesores y estudiantes, entre estudiantes y estudiantes y graduados. Salomon, Perkins y Globerson (1992) destacan los logros de la asociación entre las personas y el ordenador. Asegura que "(...) cualquier colaboración exige esfuerzo, y la colaboración intelectual entre personas y tecnología no es una excepción” (p.5). Google Drive posibilita interactuar, compartir conocimientos y reflexiones en común. Su estructura facilita la realización de trabajos colaborativos, la enseñanza personalizada y colectiva, la evaluación y autoevaluación de los alumnos.

La tecnología no es el único factor que afecta las mentes; es toda la "nube de variables correlativas" -la tecnología, la actividad, el objetivo, el ambiente, el rol del profesor, la cultura- que ejercen sus efectos combinados. En consecuencia, para manejar un efecto deseable, sea CON una tecnología inteligente o DE la misma, hace falta mucho más que la mera introducción de un nuevo programa u ordenador (Salomon et al., 1992, p.11).

En este sentido, se posibilita el aprendizaje colaborativo pues, nace y responde a un nuevo contexto sociocultural donde se define el "cómo aprendemos" (socialmente) y "dónde aprendemos" (en red) (Zañartu Correa, 2011).

Los efectos producidos CON la tecnología pueden re-definir y mejorar el rendimiento cuando los estudiantes trabajan en colaboración con las tecnologías inteligentes, esto es, aquellas que asumen una parte importante del proceso cognitivo que de otra manera correría a cargo de la persona (Salomon et al., 1992, p.11).

Desde este punto de vista, se validan las interacciones sociales, como también la visión de que el aporte de dos o más individuos que trabajan en función de una meta común, puede tener como resultado un producto más enriquecido y acabado que la propuesta de uno sólo, esto motivado por las interacciones, negociaciones y diálogos que dan origen al nuevo conocimiento. Es decir:

(...) para aprovechar al máximo la oportunidad hace falta la colaboración, no sólo entre las personas y las máquinas, sino entre personas expertas en distintos campos. Al mismo tiempo que los investigadores en el campo de la inteligencia artificial continúan desarrollando instrumentos cognitivos, los expertos en enseñanza, los investigadores de la pedagogía y el comportamiento humano, los sociólogos sensibles a los modelos de interacción cultural, e incluso los filósofos examinando cuestiones de significado y de ética, tendrán que dedicar sus opiniones y sus conocimientos al problema (Salomon et al., 1992, p.12). 
Lion (2012) asume que, "La cognición, por tanto, se entiende en un contexto de movimientos en el proceso de aprender, cuyas oportunidades se dan en todo tiempo y lugar" (p.30). Burbules (2011 en Lion 2012) señala cambios que resultan trascendentes de la vinculación entre tecnologías y aprendizajes los que son ampliados y recreados por la autora de la siguiente manera: El aprendizaje "justo a tiempo", se da cuando la gente tiene la oportunidad de aprender justo en el momento en que necesita aprender. Las ideas fuerza que priman en este tipo de aprendizaje son: relevancia, significatividad y oportunidad. En este caso la tecnología favorece procesos de transferencia cercana, con puentes a la resolución de situaciones problemáticas y contextualiza la cognición en dichas situaciones de relevancia. El aprendizaje pluricontextualizado otorga la posibilidad de aprender en todo tiempo y lugar, aprendizajes continuos y situados. El aprendizaje con otros, en colaboración, a través de redes. En este sentido, Lion (2012, p.30) señala que "Estos procesos cognitivos se inscriben en contextos que le otorgan significatividad, se comprometen en tanto situados cultural, social y políticamente".

Google Drive es una aplicación que forma parte de la plataforma gratuita Google Apps, está disponible en la nube y tiene un espacio de almacenamiento variable desde 5GB y la posibilidad de aumentar esa capacidad a 15GB si se dispone de una cuenta de gmail. Permite guardar documentos desarrollados en diferentes formatos, como en PDF. Al ser una aplicación on-line posibilita archivar en la nube, permite trabajar de manera colaborativa en grupos, compartir información, plantear dudas e hipótesis, editar un archivo entre varios integrantes (usuarios). También se puede utilizar desde diversos dispositivos móviles. Los docentes pueden coordinar y participar de los trabajos cumpliendo el rol de corrector o miembro activo del grupo.

Otra de las ventajas de almacenar la información en la nube es la protección de los archivos como pérdida o rotura del dispositivo móvil que se esté utilizando. La actualización automática se produce cuando alguno de los miembros introduce cambios en algún archivo. De este modo se incrementa el rendimiento en tiempo y trabajo asincrónico. Si los usuarios del grupo están conectados online, Google Drive permite interactuar en tiempo real, esto estimula el trabajo grupal, la presentación online entre diferentes miembros del grupo.

El Google Drive como herramienta colaborativa de enseñanza y aprendizaje, promueve la autonomía en los estudiantes y pretende superar la mirada individualista y así, lograr un verdadero aprendizaje significativo. La autora Zañartu Correa (2011) sostiene diversos principios que pretende alcanzar la mirada de la inclusión digital y el trabajo en equipo:

1) responsabilidad individual: todos los miembros son responsables de su desempeño individual dentro del grupo; 2) interdependencia positiva: los miembros del grupo deben depender los unos de los otros para lograr la meta común; 3) habilidades de colaboración: las habilidades necesarias para que el grupo funcione en forma efectiva, como el trabajo en equipo, liderazgo y solución de conflictos; 4) interacción promotora: los miembros del grupo interactúan para desarrollar relaciones interpersonales y establecer estrategias efectivas de aprendizaje; 5) proceso de grupo: el grupo reflexiona en forma periódica y evalúa su funcionamiento, efectuando los cambios necesarios para incrementar su efectividad (p.3).

La propuesta de Google Drive de la cátedra Didáctica Especial de la Geografía, cuenta con 5 (cinco) carpetas $^{5}$ con archivos. En las mismas, se encuentran los textos académicos y bibliografía de consulta correspondiente a cada unidad (eje) de la asignatura. Además, se agrega una de 'Trabajos Prácticos' a la cual los/as estudiantes suben sus producciones individuales o grupales. Asimismo, se incorpora otra que corresponde al 'Banco de Recursos', en ella se encuentran actividades y estrategias de enseñanza que han sido enriquecedoras y relevantes durante el cursado de la asignatura u otros materiales que fueron interesantes a lo largo de la carrera de profesorado.

Por otro lado, las actividades articuladas e integradas con otras asignaturas, también poseen un lugar de privilegio en el Google Drive, ya que se adiciona toda la información necesaria para integrar contenidos desde diversas asignaturas. Aquí, se encuentra la carpeta acerca de la 'Problemática del Sahel' (las materias que intervienen en la articulación son Geografía Política y Económica, Geografía de Asia y África, Didáctica Especial de la Geografía). Por su parte, se incorpora 'Pensar Malvinas' integrada por Historia Argentina Contemporánea, Geografía Política y Económica, Didáctica Especial de la Geografía. Así también, el portfolio 'Ateneo' integrado por Didáctica Especial de la Geografía y Residencia Docente, en la misma se realizan relatorías y narrativas de experiencias de las prácticas docentes. 'Memoria y enseñanza' también tiene su espacio entre los archivos, debido a que es una temática que se plantea como 5 Consideramos a las carpetas propuestas a modo de portafolio digital, ya que es una herramienta para el desarrollo profesional, que presenta múltiples usos. La intención en esta experiencia es compartir producciones, libros, normativas, actualización académica y no evaluar. Es decir es "De eminente base profesional, el portafolio digital es una herramienta que puede ser compartida, que sobrepasa los límites del aula” (Batlle Rodríguez, 2019, p.87). 
transversal, es decir todas las asignaturas tienen compromiso de abordar desde sus planificaciones la construcción de la memoria colectiva. Estas carpetas tienen la intención de promover el trabajo articulado entre las asignaturas ya que la Normativa del Ministerio de Educación de la Nación promueve este tipo de experiencias ${ }^{6}$.

Por último, se encuentra un portafolio con 'Información', en el mismo se hallan los horarios de profesores de Geografía de diversas instituciones educativas a las cuáles deben asistir, con motivo de realizar Observaciones de clases y Ayudantías en colegios de la ciudad de Santa Rosa. A partir de esta herramienta, podemos inferir en la importancia que adquiere en el proceso de formación de futuros/as profesores/as, pues supone la retroalimentación permanente, favorece el trabajo en equipo y la interacción con colegas. El intercambio de ideas y estrategias posibilita asumir un rol activo y comprometido en la enseñanza de la Geografía contemporánea.

Tal como se ha expresado, las TIC tienen un lugar imprescindible en la educación actual no solo porque son necesarias para la vida cotidiana, sino también para la profesionalización de los docentes. Es por ello que "La inclusión de las TIC en las nuevas maneras de enseñar y aprender requiere un cambio de mentalidad de los profesores y los estudiantes que, pese a su complejidad, es valorado como una transición obligatoria e irreversible en el contexto universitario actual" (Mirete Ruiz, Maquilón Sánchez, y García Sánchez, 2014, p.42).

En este proyecto, se trata de generar un espacio de aprendizajes permanente que, a pesar de la distancia real, graduados que retornan a sus ciudades natales, que no frecuentan la Universidad, la idea clave es que se sostenga la interacción. La actualización de normativas, publicaciones académicas, investigaciones, estaría al alcance de profesores que, en conjunto conforman un entorno colaborativo de formación permanente. Es decir, la intención es crear una comunidad pedagógica que comparta el interés por la enseñanza de la Geografía. Respecto al concepto de comunidad, Burbules y Callister (2006) reflexionan entorno a la propuesta de Dewey y dicen que "su definición de la comunidad se funda en la idea de la identificación (o la creación) de características comunes que puedan sostener un sentido de cohesión" (p.252). En síntesis, la idea es “(...) generar prácticas pedagógicas que alcancen los entornos tecnológicos en los que vivimos" (Maggio, 2018, p.146). Nuestra propuesta consiste en formar una comunidad cuyos intereses compartidos por la enseñanza de la Geografía, sostenga un espacio de encuentro y actualización que responda a las coordenadas de la época.

\section{Reflexiones}

Crear y poner a disposición de los/as estudiantes espacios compartidos de producción de conocimientos a través del uso de tecnologías se constituye como clave pedagógica para facilitar el proceso de formación permanente y continua de los/as profesores/as. En este sentido, el uso de Google Drive como dispositivo de formación resulta significativo para lograr el acercamiento a la Universidad y la actualización académica de profesores noveles y futuros profesores/as en Geografía que cursan la materia Didáctica Especial de Geografía de la que forma parte este proyecto. Ello implica ampliar los tiempos y los espacios de trabajo, participación física en el aula y la cursada de la asignatura a partir de constituir comunidades pedagógicas de aprendizajes. Bajo las premisas de cooperación y colaboración se propone un esquema organizativo para intercambiar y enriquecer el trabajo profesional docente en el que la tecnología se convierte en mediadora de este proceso.

En ese sentido, las/os profesoras/es universitarios/as adquieren un rol central en la mediación entre el conocimiento disciplinar y el conocimiento tecnológico, pues establecen un puente de comunicación virtual entre estudiantes avanzados/as y profesores/as. El desafío no sólo se piensa en función de recuperar y acercar a aquellos/as docentes que se encuentran alejados del ámbito universitario y mantener activos a los/as estudiantes de grado, sino colocarlos/as en relación a las estrategias de enseñanza en los nuevos contextos y escenarios educativos que implican, por un lado, lograr mejorar las prácticas de enseñanza y por otro, apropiarse de las tecnologías para propiciar inclusión educativa y tecnológica.

\footnotetext{
6 Resolución CFE $\mathrm{N}^{\circ}$ 93/09. Asimismo, en relación a la necesidad de recuperar evidencias basadas en la innovación de propuestas, el Ministerio de Educación de la Provincia de La Pampa, en el año 2019, realizó un encuentro con instituciones educativas a partir de un itinerario basado en prácticas pedagógicas y reconstrucciones de los escenarios escolares, denominado "Innovar Educa 2019". Esta iniciativa permitió indagar acerca de propuestas de enseñanza que implican una resignificación de las experiencias escolares en contexto. Existen propuestas que promueven múltiples posibilidades de enseñanza y de aprendizaje para nuestros estudiantes, es decir, oportunidades para la formación y el desarrollo profesional continuo de nuestra comunidad educativa. Uno de los ejes que se abordaron fueron las Tecnologías digitales en la enseñanza y la gestión.
} 


\section{Referencias bibliográficas}

Acosta, M. y Pérez, G. (2019). Las TIC en las clases de Geografía. Desafíos para la enseñanza en contextos de inclusión educativa. En M. C. Nin, (Comp.), Geografía y enseñanza. Investigación, reflexiones y prácticas (pp. 73-92). Colección Libros Académicos de Interés Regional. Santa Rosa: EdUNLPam.

Batle Rodríguez, J. (2019). El portafolio digital en acción: posibilidades para su puesta en práctica. En Pujolà, J-T. (2019). El Portafolio digital en la docencia Universitaria. Barcelona: Octaedro.

Benejam, P. (2001). Los contenidos de la Didáctica de las Ciencias Sociales en la Formación del Profesorado. En C. Arrondo y S. Bembo. (Comp.), La formación docente en el profesorado de Historia (pp. 61-70), Rosario, Argentina: Homo Sapiens.

Buckingham, D. (2008). Más allá de la tecnología. Buenos Aires: Manantial.

Burbules, N. y Callister, T. (2006). Educación: riesgos y Promesas de las Nuevas tecnologías de la información. Buenos Aires: Gránica.

Castells, M. (2008). La era de la información. Economía, Sociedad y Cultura, Vol. 1. La Sociedad Red. México: Siglo XXI editores.

Comisión Económica para América Latina y el Caribe. (2008). Claves para la innovación social en América latina y el Caribe. Chile: Publicación de Naciones Unidas.

Coronado, M. (2013). Competencias Docentes. Ampliación, enriquecimiento y consolidación de la práctica profesional. Buenos Aires: Noveduc.

de Souza Cavalcanti, L. y Dos Santos, L. (2020). Formar para la docencia en geografía: un camino para la cooperación. Didáctica Geográfica, 21, 2020, pp. 19-39. doi: https://doi.org/10.21138/DG.471

Lion, C. (2012). Pensar en red. Metáforas y escenarios. En A. Scialabba y M. Narodowski (Comp.). ¿Cómo serán? El futuro de la escuela y las nuevas tecnologías. Buenos Aires: Prometeo.

Lyotard, J. F. (1994). La Condición Postmoderna. Madrid: Cátedra.

Litwin, E. (2005). Tecnología educativa: política, historias, propuestas. Buenos Aires: Paidós.

Maggio, M. (2018). Reinventar la clase en la Universidad. Buenos Aires: Paidós.

Maggio, M. (2012). Enriquecer la enseñanza. Los ambientes con alta disposición tecnológica como oportunidad. Buenos Aires: Paidós.

Manso, M., Pérez, P., Libedinsky, M., Light, D. y Garzón, M. (2011). Las TIC en las aulas. Experiencias latinoamericanas. Buenos Aires: Paidós.

Ministerio de Educación de la Nación (2006). Ley de Educación Nacional (LEN) № 26.206.

Ministerio de Educación de La Pampa (2020). Resolución № 701/20. Criterios de evaluación, acreditación y promoción para los/as estudiantes que se encuentren cursando su escolaridad de manera presencial, no presencial o alternada del Sistema Educativo Provincial. Recuperado de: https:// repositorio.lapampa.edu.ar/index.php/normativa/item/resolucion-n-701-20

Mirete Ruiz, A. B., Maquilón, J.J. y García Sánchez, F.A. (2014). Enfoques de Aprendizaje y Tic en Educación Superior. Colección: Proceedings del Foro Internacional sobre Evaluación de la Calidad de la Investigación y la Educación Superior, pp. 41-46. Recuperado de: https://www.researchgate.net/ publication/320558796_ENFOQUES_DE_APRENDIZAJE_Y_TIC_EN_EDUCACION_SUPERIOR

Murillo,L.Y.(2011).Didáctica de lageografíay lasnuevastecnologías(Tesis doctoral).Universitat Rovira IVirgili, Tarragona, España. [Versión electrónica]: http://www.tdx.cat/bitstream/handle/10803/42931/ TESI.pdf;jsessionid=45153736C1A33E53BA56263C0AF94F9A.tdx2? sequence $=1$

Nin, M. C. \& Acosta, M. I. (2020). Prácticas evaluativas en la enseñanza de la geografía en educación secundaria. Boletín geográfico, 42(1), 83-102. Recuperado de: http://revele.uncoma.edu.ar/htdoc/ revele/index.php/geografia/article/view/2703/59523

Nin, M. C.; Acosta, M. I. y Leduc, S. M. (2020). Pandemia en el sigo XXI. Reflexiones de la(s) geografía(s) para su comprensión y enseñanza. Revista Huellas, 24(1) (2020). doi: http://dx.doi.org/10.19137/huellas

Nin, M. C.; Leduc, S. M. y Acosta, M. I. (2020). La planificación como objeto de investigación en la formación docente en Geografía. Revista REIDICS, 6 pp. 94-111. doi: https://doi.org/10.17398/2531-0968.06.94

Perrenoud, P. (2007). Diez nuevas competencias para enseñar. Barcelona: Grao. 
Poggi, M. (2007). Prólogo. En C. Magadán y V. Kelly (Comp.). Las TIC. Del aula a la agenda política (pp. 16-18). Buenos Aires: UNICEF.

Pozo Sánchez, S., López Belmonte, J., Moreno Guerrero, A. J. e Hinojo Lucena, F. J. (2020). Flipped learning y competencia digital: Una conexión docente necesaria para su desarrollo en la educación actual. Revista electrónica Interuniversitaria de Formación del Profesorado, 23(2), 127-141. doi: https://doi. org/10.6018/reifop.422971

Salomon, G., Perkins, D. y Globerson, T. (1992). Coparticipando en el conocimiento: la ampliación de la inteligencia humana con las tecnologías inteligentes. Revista Comunicación, lenguaje y educación. (13), 6-22.

Siemens, G. (2004). Conectivismo: Una teoría de aprendizaje para la era digital. P. 1-10. Traducción Diego Leal Fonseca 2007. Recuperado de: https://www.comenius.cl/recursos/virtual/minsal_v2/ Modulo_1/Recursos/Lectura/conectivismo_Siemens.pdf

Souto González, X. (1999). Didáctica de la Geografía. Problemas sociales y Conocimiento del Medio. Barcelona: Ediciones del Serbal.

Tedesco, J. C. (2007). Las TIC en la agenda política educativa. En C. Magadán y V. Kelly (Comp.), Las TIC. Del aula a la agenda política (pp. 25-30). Buenos Aires: UNICEF. Recuperado de: https://es.slideshare. net/indudable/las-tic-del-aula-a-la-agenda-poltica-memorias-seminario-argentina

Zañartu Correa, L. M. (2011). Aprendizaje colaborativo: una nueva forma de diálogo interpersonal y en red. Revista digital de educación y nuevas tecnologías: Contexto educativo, 28. pp. 1-10. Recuperado de: http://files.enriquecer-educaciontic.webnode.es/200000026-9a1009c069/Contexto_Educativo_-_ Revista_digital_de_Educacion_y_Nuevas_Tecnologias.pdf 